\title{
Microfiltración marginal en restauraciones con amalgama. Revisión
}

\author{
Marginal microfiltration in amalgam restorations. Review
}

\section{Resumen}

El presente artículo es un revisión bibliográfica del fenómeno de microfiltración en restauraciones con amalgama y sus consecuencias tales omo cambio de coloración en la interfase diente-restauración, delerioro marginal, semsibilidad dentinaria postoperatoria, caries secundaria y la inflamación pulpar.

Además se mencionan los mecanismos para reducir la microfiltración y sus efectos mediante el uso de sellantes dentinarios representados por los barnices cavitarios y los sistemas adhesivos.

Concluye señalando que la amalgama permanece como el material de restauración mas usado en el sector posterior. Asimismo enfatiza que cuando el tamaño de la brecha interfase diente-amalgama es relativamente grande, es posible que se presente una marcada sensibilidad post-operatoria

\section{Abstract}

The present articule is review references from phenomenon of microfiltration in restorations with amalgam and yours consecuents in changes of color in the interface touth-restorations,margin deterioted, sensitivity dentinarea postoperate, caries secondary and pulp inflamation.

Besides naming the mechanicals for to reduce microfiltration, and yours effects for use of sealers dentinaries representation for the varnish cavitys and adhesive systems.

Conclusive indicate wath the amalgam is the materials to most use in the back sector.

Also indicate walh whem the very large of the opening interface tooth -amalgam is relative big is possibily to present pronunced postoperative sensitivity

\section{Introducción}

La amalgama dental ha sido criticada en lo que respecta al efecto del mercurio en la salud humana y el impacto de su uso en el ambiente.

A pesar de la campaña anti-amalgama y el incremento en la demanda de restauraciones estéticas, la amalgama dental permanece como el material de restauración mas comúnmente utilizado en restauraciones de tamaño moderado y grande en el sector posterior.

Unla encuesta reciente a mas de 8,000 odontólogos demostró que la amalgama es seleccionada antes que las resinas compuestas en una relación de 4:1 para restauraciones clase II en dientes temporales y permanentes (Clinical Research Associates, 1995)].

La amalgama sigue siendo el material por excelencia para realizar restauraciones en el seclor posterior, su técnica es poco sensitiva y de bajo costo, tiene buena longevidad y además ro existe evidencia clínica que pruebe que haya una relación entre la presencia de restauraciones de amalgama y problemas de salud .

A causa de su aparente facilidad de manipulación muchos odontólogos descuidan su técnica operatoria . En una estadistica efectuada sobre 654 dientes portadores de restauraciones de amalgama, se hallo que el $10 \%$ eran correctas, el $20 \%$ se considero aceptable y el $70 \%$ eran deficientes.

WING G. ${ }^{2}$ en un examen practicado sobre 100 dientes que tenían obturaciones de amalgama, comprobo que el 875 de los casos denotaban deficiente preparación cavitaria, el $89 \%$ fallas en la reconstrucción anatomica, el $90 \%$ incorrecta terminación y el $62 \%$ mala condensación

Uno de los problemas asociados con las restauraciones de amalgama es la microfiltración a lo largo de la interfase diente-restauración, sin embargo la amalgama es capaz de impedir la microfiltración a través de los productos de corrosión que sellan
ARTíClLo DE Revisión

\section{Víctor Lahoud Salem"}

Departamento Académico de Estomatología Rehabilitadora

* Facultad de Odontología, Universidad Nacional Mayor de San Marcos, I ima, Perú E-mail: vlahouds@unmsm.edu.pe

Palabras clave: microfiltración. Selladores cavitarios. Adhesivo

Key words: microfiltration. Sealers cavitys.Adhesive la interfase Taring $\mathbf{B}^{3}$, La filtración marginal es mayor en la amalgama recien colocada y se reduce con el tiempo debido a los productos de corrosion que se depositan en la interfase diente-restauración y produce un autosellado.

\section{Discusión}

Going $\mathrm{R}^{4}$, señala que formación de brechas en el margen cavo-superficial trae como consecuencia microfiltración marginal, que es la responsable de los cambios de coloración en la interfase diente-restauración, la ruptura marginal, la sensibilidad dentinaria, la caries recidivante y la inflamación pulpar. Mahler $D^{5}$, afirma que la brecha entre el material restaurador y el diente es el resultado de diversas variables, que incluyen, la falta de adhesión química, la diferencia de coeficiente de expansión térmica, los cambios dimensionales durante el endurecimiento de la amalgama, la ina decuada condensación y adaptación 
a las paredes cavitarias y la inadecuada relación aleación mercurio.

Cabe resaltar la sensibilidad postopertoria relacionada con la microfiltración. La Teoría hidrodinámica de Brannstrom y Astrom A. ${ }^{6}$, ofreció una explicación para esle fenómeno. Esta teoría dice que la brecha a nivel de la interfase amalgamadiente se llena con el fluido proveniente de los canalículos inmediatamente después de colocar la restauración, el movimiento y los cambios de presión de dicho lluido causa la excitación de terminaciones nerviosas de la pulpa. Un estímulo térmico puede exacerbar esta respuesta cambiando el volumen y la presión del fluido de los canalículos en la brecha. Cuando el tamaño de la brecha es relativamente grande se incrementa la sensibilidad post-operatoria Mahler y Nelson?

Entre los mecanismos para reducir la microfiltración, la disminución de la sensibilidad post-operatoria y la disminución de la caries recidivantes exislen: los selladores dentinarios que están representados por los barnices cavitarios y los sistemas adhesivos. Geddes ${ }^{8}{ }^{8}$, Hilton $T^{9}$, con ellos se logra una película protectora de poco espesor, por lo que no actúan como aislantes térmicos, sino como una barrera que reduce la sensibilidad post-operatoria y la microfiltración marginal, Phillips $\mathrm{R}^{10}$
Neme $A^{11}$, afirma que la aplicación de los sistemas adhesivos tienen por finalidad lograr el sellado marginal y la retención del material restaurador.

Los materiales adhesivos que se usan para lograr la unión de la amalgama a la estructura dentaria, se denominan adhesivos para amalgama

\section{Conclusiones}

1.- $\mathrm{El}$ uso de los adhesivos modernos debajo de las restauraciones de amalgama producen un sellado inmediato entre la interfase dienterestauración.

2.- La adhesión entre la amalgama y el adhesivo es de carácter mecánico y esta formada por la interdigitación del adhesivo que se protege dentro de la amalgama

3.-I )iferentes estudios in vitro para evaluar la microfiltración, coinciden en que los sistemas adhesivos utilizados debajo de restauraciones de amalgama reducen efectivamente la microfiltración.

\section{Referencias}

1.Clinical Research Associates: Product use survey clinical Research Associates Newsletter. 1995, 19(10): 4

2.Wing,G.: Modern Conceptsfor the amalgam restration.Dont Clinin $\mathrm{N}$ Amer.1991, 15: 43-56
3.Taring B.: Marginal Integrity of bonded amalgam restorations Am J Dent. 1996, 9(2):72-76

4.Going R.: Microleakage around dental restorations.J .Am Dent Assoc. 1972, 84:13499-1357.

5.Mahler D.: One Year Clinical Evaluation of bonded amalgam restorations. Journal the American Dental Association. 1996, 127:345349.

6. Brannstrom $M$ \& Astrom A.: The hidrodinamics of the dentine, it possible relationship to dentinal pain. internacional Dental Journal. 1972, 22:219- 227.

7.Mahler D. Nelson L.: Factor affecting the marginal leakage of amalgam Journal the American Associaton. 1984, 108:51-54

8.Geddes I..: Proteccion dentino pulpar. Fn Mooney B Operatoria Dental $3^{\mathrm{a}}$ ed. Buenos Aires. Editorial MédicoPanamericana. 1999:692-719.

9.Hilton T.: Cavity sealers,liners, and bases oper dent. 1996, 21:134- 146

10.Phillps R.: La Ciencia de los Materiales Dentales $9^{\star}$ ed. México Interamericana. 1993.

11.Neme A.: Evaluation of dental adhesive systems with amalgam and resin composite restorations Oper. Dent. 2000, 25:512-519. 